\title{
Retail marketing para desarrollar mercados emergentes
}

\author{
José Antonio Taquía Gutiérrez \\ Universidad de Lima. Lima, Perú \\ Correo electrónico: Jtaquia@correo.ulima.edu.pe
}

Recibido: 21 de abril de 2015 / Aprobado: 25 de julio de 2015

\begin{abstract}
Resumen: Para una empresa es muy alto el riesgo de tomar decisiones comerciales sin una adecuada evaluación de sus efectos. Para evitarlo, se requiere de métodos y herramientas de análisis que vinculen el proceso comercial y el proceso de abastecimiento, buscando que no se produzca exceso de inventario o que no se reduzca la exposición a perder volumen en el mercado por falta de productos en el punto de venta. Consideramos que algunas técnicas de ingeniería de operaciones presentadas en este artículo pueden ayudar a que el crecimiento retail genere un impacto positivo en el desarrollo del mercado y la ciudad.
\end{abstract}

Palabras clave: cadena de abastecimiento / marketing / retail marketing / gestión de categoría / riesgo en decisiones / ciclo de vida de productos / comercio minorista

\section{Retail Marketing to Develop Emerging Markets}

Abstract: Business decisions require adequate evaluation of its effects. To reduce involved risk on them, we bold the importance of analyze the link between the commercial and the provisioning process in order to grow without unnecessary inventory or reduce exposure to lose volume in the market for lack of products at the point of sale. We believe that some engineering techniques presented in this article can help to make a positive impact of increasing retail operations on the market and city development.

Key words: supply chain / tactical marketing / marketing / category management / risk decisions / products life cycle / retail 


\section{INTRODUCCIÓN}

Es indudable que el crecimiento de la ciudad de Lima y el canal retail se mantendrán en los próximos años. Al mismo tiempo, los efectos de un crecimiento desordenado de la ciudad se reflejan en problemas estructurales de tráfico, inseguridad y calidad de vida. ¿Cuál será el impacto de este desorden en el crecimiento del sector retail en la capital peruana? ¿Crecerá ordenadamente en este entorno? ¿Servirán las técnicas de ingeniería para hacer más eficientes las operaciones de comercialización y distribución en estos mercados, ya de por sí estructuralmente complejos? Este crecimiento urbanístico representa todo un reto para las empresas retail que incrementarán sus inversiones en marketing y distribución, debido a la apertura de nuevos puntos y canales de ventas. Los diferentes formatos de tiendas de gran superficie $\left(5000\right.$ a $\left.6000 \mathrm{~m}^{2}\right)$ al inicio de los años noventa están cambiando a formatos de menor tamaño, debido a la falta de terrenos y a la saturación de zonas con alta densidad poblacional. Por este motivo, el ámbito geográfico foco del presente artículo, Lima este, es una gran interrogante, si consideramos que comprende los distritos de Ate, El Agustino, San Juan de Lurigancho y Santa Anita. Este grupo de distritos representa el 25,6\% del total de puntos de comercio y bodegas en todo Lima (Ipsos, 2013).

En San Juan de Lurigancho, donde se calcula que viven casi un millón de habitantes, solo existen ocho supermercados repartidos entre dos competidores en las zonas más próximas al ingreso del distrito. Entre sus límites están los distritos de Carabayllo, El Agustino, Lima, Rímac, Independencia y Comas. Su ubicación es estratégica para cualquier emprendimiento o empresa que desee crecer en la región Lima y la movilidad de los consumidores ha llevado a que San Juan de Lurigancho posea un potencial similar al que mostraba Villa El Salvador en los años ochenta, el cual hoy es un polo industrial de Lima. Otro factor relevante de su proyección comercial es que su población es mayoritariamente joven.

El impulso que traen las inversiones en San Juan de Lurigancho en temas como el transporte en tren eléctrico ha generado una mayor dinámica comercial, debido a la movilidad de los clientes y la facilidad para acceder a este distrito. Actualmente se calcula que trasladarse desde San Juan de Lurigancho hacia Lima y Villa El Salvador puede demorar 20 y 45 minutos, respectivamente.

Con relación a la evolución del mercado, podemos decir que el canal tradicional continúa siendo el más representativo, pero que el canal 
Callao

51 Bellavista

52 Callao

53 Carmen de la Legua

54 La Perla

55 La Punta

56 Ventanilla

\section{Lima centro}

05 Breña

13 La Victoria

01 Lima (Cercado)

25 Rímac

30 San Luis

Lima moderna

04 Barranco

11Jesús María

14 Lince

17 Magdalena del Mar

18 Miraflores

21 Pueblo Libre

27 San Isidro

32 San Miguel

34 Surquillo

12 La Molina

41 San Borja

33 Santiago de Surco

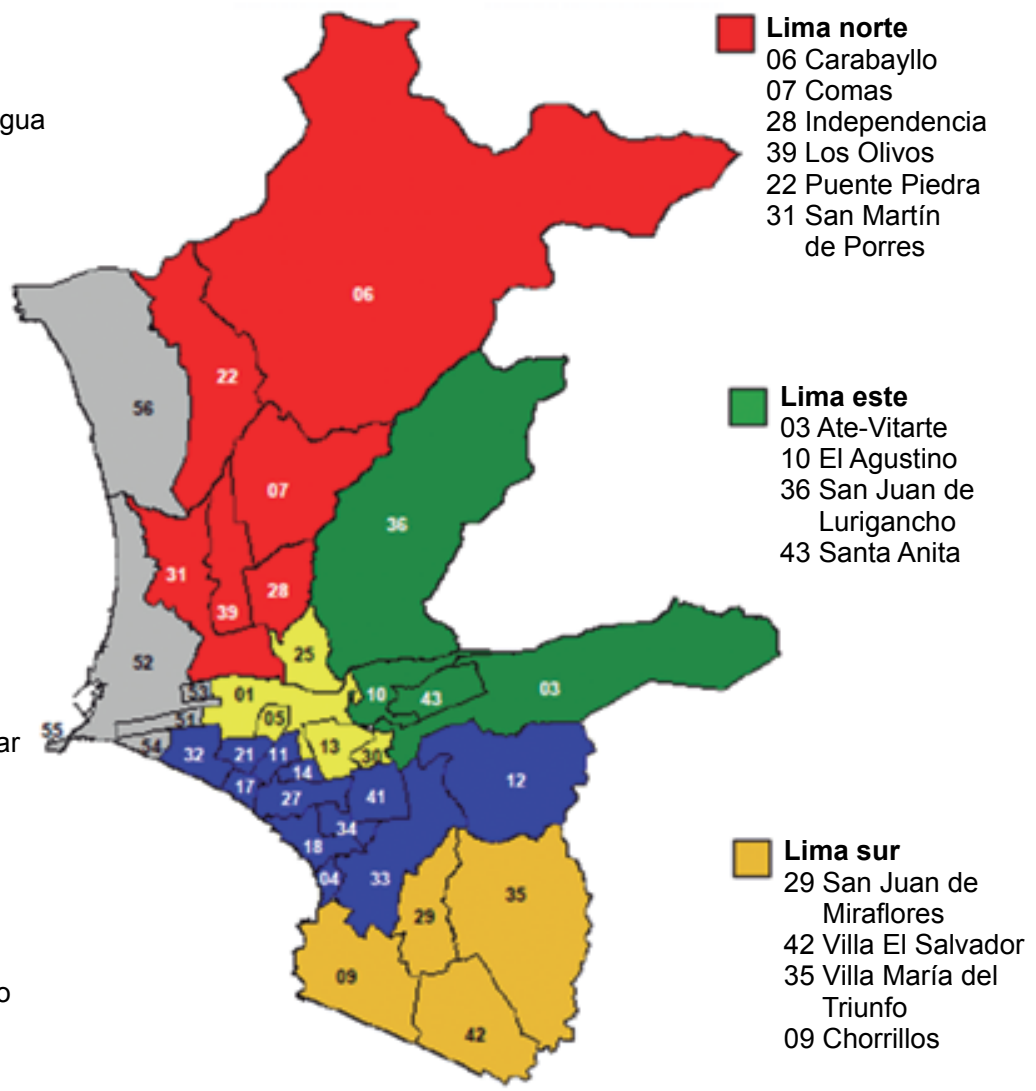

Figura 1. Segmentación de Lima metropolitana

Fuente: Ipsos Apoyo. Estudio canal tradicional (2013)

moderno viene creciendo y diseñando sus ofertas, tomando en cuenta que el público consumidor planifica sus compras; el $70 \%$ pasa su tiempo recorriendo el negocio y observando porque está más actualizado, el $73 \%$ aprovecha las promociones, y a más del $50 \%$ le gusta probar productos nuevos. En esta experiencia de compra el precio es una variable muy importante, la cual debe ser comunicada adecuadamente.

En este contexto, el canal moderno se encuentra en el camino de la evolución, y todo está en manos de las nuevas estrategias que utilicen las empresas del sector para atraer a más consumidores, fidelizarlos y asegurar un mayor crecimiento en el consumo y desembolso del hogar. Esto también explica que el fenómeno social del uso intensivo de medios electrónicos ha generado nuevas maneras de comunicarnos y so- 
cializar, modificando los procesos de toma de decisiones para comprar en entornos retail y en la manera de entregarlos por parte de la cadena de abastecimiento.

Esta dinámica del mercado requiere identificar la convivencia de productos con enfoque en el sistema pull-push (Singh y Raghuvanshi, 2014) y su impacto en la función de riesgo de venta perdida. Ambos sistemas requieren enfoques distintos de gestión de la categoría y de espacio en el local. Asimismo, el perfil del consumidor de supermercados de Lima este, en especial de San Juan de Lurigancho, debe incorporar criterios de experiencia de compra diferentes al de otras zonas geográficas de Lima. Para acompañar el crecimiento del canal retail en este mercado emergente se requiere estructurar las categorías, implementar mecanismos de consensos de ventas en valor y volumen, estructurar el canal de distribución y las tácticas de abastecimiento eficientes. Elementos que deben estar dentro del plan comercial para desarrollar el mercado. A la vez que orientada a las autoridades ediles y de tránsito, esta información está directamente vinculada con la periodicidad de abastecimiento y su impacto en el tráfico de la zona donde se abran locales o grandes centros comerciales.

\section{ESTRUCTURACIÓN DE UNA CATEGORÍA}

Uno de los pilares del crecimiento retail es la gestión de la categoría comercial. Esta capacidad de gestión táctica influye en toda la cadena de abastecimiento, en especial desarrollando procesos de coordinación a través de los cuales los proveedores y las empresas del canal retail formulan planes conjuntos de abastecimiento (Kurtulus y Toktay, 2012).

Debido al proceso de transformación profundo del canal moderno ante un cliente más exigente, se requieren nuevas técnicas de pronósticos de ventas, como las series de tiempo probabilísticas, regresión bayesiana, entre otras (Ntzoufras, 2011), porque la información histórica útil para proyectar será cada vez menor, debido a la propia dinámica de estos cambios.

En el caso de los arreglos florales, las fechas del Día de la Amistad o del Día de la Madre representan el mayor ingreso por ventas para las empresas retail del rubro; asimismo, en el caso del consumo de pavo en el país, la fecha previa a fiestas navideñas representa más del $50 \%$ de lo vendido anualmente; es un producto que tiene un ciclo de consumo 
de horas y una operación de varios meses previos de preparación y beneficio. Diseñar una logística de entrada y salida para un producto perecible requiere de tácticas para estandarizar los pedidos en formatos únicos. Eso impide exponerse a un exceso en el inventario. Lo mismo ocurre con los productos menos estacionales, como los de decoración para los hogares en el caso de Casa \& Ideas (Crespo, 2015).

En muchos casos los productos son abastecidos por proveedores de China o de Europa, o son productos con mayor rotación que otros, por lo que el responsable de gestionar la categoría debe considerar atraer un mayor flujo de visitantes a la tienda mediante promociones especiales, con el fin de generar tráfico y exponer al lado de dichas ofertas productos de mayor margen, para balancear el retorno de la inversión.

\subsection{Gestión de categorías dinámicas}

Una categoría dinámica es aquella que gestiona la convivencia en su portafolio de productos con ciclos de vida diferentes frente al cliente y al período de tiempo esperado, según su curva de agotamiento en inventario. No existen categorías que puedan desarrollarse sin una adecuada visión sistémica de cómo conviven entre sí los productos y cómo se van traslapando, en función de las diversas actividades de marketing y promoción, en el pareto de las ventas o las listas top de productos de una campaña.

Como lo menciona Labajo (2007), existen productos que por su naturaleza se pueden clasificar en:

- Categorías de destino. Son las consideradas como artículos de necesidad básica, y donde la decisión de quien compra el producto se basa en el precio.

- Categorías de rutina. Son aquellas que el consumidor compra regularmente en la tienda, como leche, café o detergente, debido a factores como cercanía, variedad, etc.

- Ocasionales o estacionales. Son aquellas adquiridas con menor frecuencia, como helados o protector solar.

- Categorías de conveniencia. Son aquellas en las que el consumidor sabe que puede encontrar una selección mayor a menor precio en otro lugar, como gaseosas, sopas o enlatados, pero ese mayor valor no es suficiente como para visitar otra tienda. 
Asimismo, por el lado de la exposición del producto en la sala de ventas, encontramos que la mezcla puede considerarse: agresiva, diferenciada, competitiva y de velocidad.

Tomando como ejemplo las ventas en el mercado de venta retail directo, un mix agresivo es aquel que incluye productos con alta percepción en los clientes y con buena rotación de inventario. Usualmente se aplica en periodos de venta claves para el negocio; por ejemplo, en el mercado de cosméticos, en los meses del Día de la Madre o de las fiestas navideñas.

La oferta del mix diferenciado se da en el caso de productos que tienen, por algún atributo, la ventaja de la diferenciación; puede ser una mejor formulación o una presentación diferente y atractiva frente a los productos competidores. En este tipo de mix es donde se busca mejorar el margen de la empresa, porque el cliente persigue más el beneficio que el ahorro en el precio.

En general, es la marca líder del mercado la que tiene mayor poder de negociación frente al canal retail buscando mantener ese liderazgo, ya sea negociando volúmenes de consumo y ubicación preferente en el patio de ventas.

Se considera una buena práctica estudiar las decisiones de compra (Díaz, 2000), así como el análisis conjunto de productos para ver la intención de compra a diversos precios; ambos métodos son muy útiles cuando la categoría tiene demasiada variedad y complejidad por la cantidad de competidores. Posteriormente, una vez conocida la demanda de los productos por tienda, se buscará gestionar una categoría. Esto último tiene aún mucho campo por desarrollar en el mercado peruano.

También se debe tener en cuenta la venta cruzada entre productos de una categoría o entre segmentos de esta. Para ello se requiere información histórica de ventas y unidades, y mediante el uso de la prueba estadística de correlación de Pearson se pueden identificar cruces entre los productos analizados. También es interesante conocer el índice de saturación del canal de venta, tomando como referencia el efecto de descuentos consecutivos y la variación en el consumo ante una misma oferta utilizada de manera consecutiva.

\subsection{Lanzamiento de productos nuevos}

El escenario natural de un producto nuevo es atender una necesidad también nueva del mercado y desarrollar procesos de abastecimiento 
que probablemente no hayan existido previamente. Pero también podemos referirnos a productos nuevos como extensiones de línea, relanzamientos de productos o al incremento de alguna funcionalidad que el área de marketing desea mostrar al mercado para diferenciarse e incrementar su participación. En cualquiera de estos casos el producto recién introducido se convertirá en un nuevo código de operación (stock keeping unit), que lo identificará a lo largo de su ciclo de vida comercial.

Como es un producto sin historia se debe preparar la base de información que nos servirá para cuantificar su desarrollo futuro. En estos lanzamientos el área de marketing debe diseñar una estrategia de 360 grados sobre las variables de demostración, difusión e inversión asignada a la promoción que permitan al equipo que debe cuantificar el volumen de proyección encontrar alguna tendencia que posibilite una alta eficiencia de la asignación de recursos del lanzamiento (Jeyaraj y Sabherwal, 2014). Por el lado de la organización de operaciones, toda la información del posicionamiento de dicho producto en el rango del portafolio de la categoría representa información valiosa para coordinar con los proveedores los tamaños de los lotes y los tipos de abastecimiento, en función al plan de salida del producto en caso de que tuviera que atenderse diversos mercados geográficos.

La presencia, en este proceso, de un panel de expertos que con su experiencia sugieran acciones para establecer un plan adecuado es muy

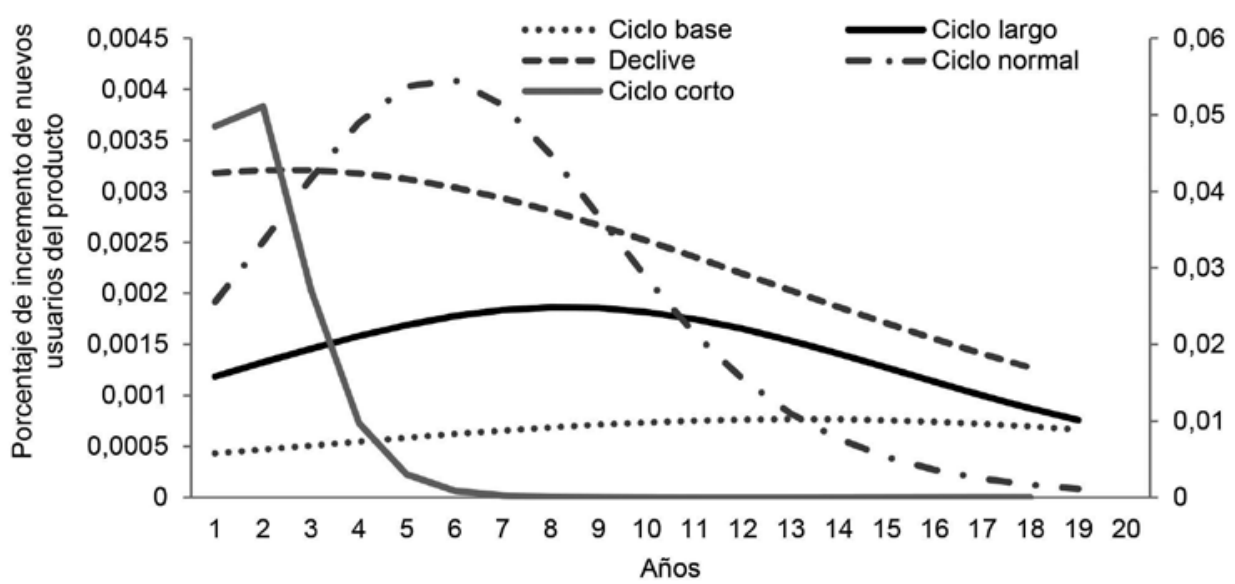

Figura 2. Curvas de ciclo de vida: productos en categoría Elaboración propia 
relevante. Cada negocio tiene su propia dinámica de demanda y una categoría siempre debe visualizarse como parte de un todo. Este método de panel de expertos usualmente se denomina método Delphi, el cual recoge una combinación de opiniones que pueden establecer picos de máximos y mínimos, que ayudarán a limitar la exposición al riesgo ante la incertidumbre que se genera durante la preparación de un lanzamiento.

Después de haber cubierto todas las dimensiones subjetivas internas y externas del impacto de incorporar un producto nuevo dentro de la categoría, se debe buscar la historia de comportamiento de productos similares al que se desea lanzar. Esta semejanza se puede dar por perfil de consumidores objetivo, historia de unidades vendidas de estos productos, información primaria y secundaria del rango de precios y la elasticidad en el punto de venta de dichos productos.

Usualmente se busca un producto muy similar al que se desea analizar. La experiencia en la investigación nos muestra que lo que se utiliza es una combinación ponderada de varios productos que pueden aportar base, ruido y efecto al nuevo producto. Se sugieren entre tres o cuatro productos históricos. Estos también pueden ser propuestos por el panel de expertos. Se entiende como base a las unidades o ventas por pura necesidad del producto sin esfuerzo promocional. El ruido hace mención a la distorsión, pudiendo basarse en la raíz media cuadrática del error (RMSE, por sus siglas en inglés) que establecen picos de variación, y por último el efecto, que lo da la labor de estrategia en el precio oferta y que estimulan la demanda, el cual debe ocurrir con periodicidad según las variables de estacionalidad o momento de cada negocio.

Para modelar una curva de ciclo de vida del producto nuevo se utilizan diversas funciones, como Gompertz, Bass, o la curva logística. La idea es construir una curva en forma de S. Estas se nutren en su fundamento de la función generatriz Weibull, que emplea tres parámetros. Se pueden establecer diversas posibilidades para alcanzar el máximo potencial de la función y en cada etapa de la curva se requiere de supuestos y coordinaciones para influenciar en el mercado para que el lanzamiento logre dicho comportamiento.

Muchas industrias, en especial las vinculadas a la moda, utilizan prelanzamientos y pruebas piloto para medir el nivel de aceptación de dicha presentación. En esta etapa los resultados pueden incorporarse al modelo, buscando ajustar sus parámetros, pues es una retroalimentación válida de lo que puede ocurrir en el total del mercado objetivo. 


\section{GESTIÓN DE RIESGO OPERATIVO EN UNA CATEGORÍA}

Podemos considerar que la gestión de riesgo es la cultura, procesos y estructuras orientadas a identificar oportunidades y reducir o eliminar los efectos adversos en el entorno de una organización. Es una aproximación al análisis predictivo bajo un enfoque de procesos. En la presente investigación el riesgo está enfocado al proceso de abastecimiento de productos con mucha incertidumbre en su demanda, por lo que debemos encontrar alguna métrica que nos permita configurar un escenario del proceso (Wieland y Wallenburg, 2012). Como la gestión del abastecimiento es consecuencia de la medición del pronóstico de demanda, utilizaremos la medición del pronóstico futuro como una fuente de información que, gestionada con un enfoque predictivo, se convierte en una herramienta táctica para atenuar las naturales fluctuaciones del proceso de estimación. Existe material referencial que aborda este tipo de enfoque: Gaur, Kesavan, Raman y Fisher (2007), Fildes y Kingsman (2011) y Chase (2013), pero hay diferencias en la práctica local de aspectos vitales debido a que en el mercado retail peruano hay muy poco uso de técnicas cuantitativas para estimar la demanda y como consecuencia de esta definición establecer una periodicidad adecuada de abastecimiento.

Como parte del proceso de investigación, se ha buscado enfocar la volatilidad de la demanda del mercado como el origen de la incertidumbre que, cuantificada, nos dará el riesgo del abastecimiento, ya sea que se exprese como inventario excedente o faltante. En todo proceso de gestión del riesgo la primera etapa consiste en identificar los riesgos a los que está expuesto el proceso; estos riesgos pueden generar demoras o deteriorar sistemáticamente sus resultados.

Después de haber identificado los riesgos, estos deben ser evaluados a través de métricas definidas, que mantengan los resultados dentro de los niveles esperados. Todo este esfuerzo debe ir acompañado por actividades de comunicación y difusión de los hallazgos en relación con la gestión del riesgo para generar una cultura orientada a su mitigación.

La mitigación del riesgo puede realizarse de varias maneras: evitar totalmente el riesgo, prevenir y controlarlo, retener el riesgo y transferir el riesgo a través de la protección, el aseguramiento y la diversificación. Estas son estrategias genéricas, que enmarcan diversas formas de aplicación y que varían según el segmento del mercado retail ana- 
lizado: financiero (bancos y cajas de ahorro), de salud (productos médicos), servicios (cuidado personal, gimnasios, etc.) o comercialización de productos no perecibles y perecibles (mercado moderno en todos sus formatos, como son hiper, super y minimercado).

Tabla 1

Variabilidad en los componentes del sistema de abastecimiento

\begin{tabular}{|c|c|c|}
\hline \multicolumn{3}{|c|}{$\begin{array}{c}\text { Influencia de la variabilidad de los componentes estructurales } \\
\text { en el sistema de gestión de abastecimiento }\end{array}$} \\
\hline $\begin{array}{l}\text { Origen en los } \\
\text { proveedores }\end{array}$ & $\begin{array}{l}\text { Foco en la gestión de } \\
\text { proveedores }\end{array}$ & $\begin{array}{l}\text { Inestabilidad en los tiempos de } \\
\text { abastecimiento }\end{array}$ \\
\hline \multirow{7}{*}{ Origen interno } & \multirow{5}{*}{$\begin{array}{l}\text { Relacionado con } \\
\text { la manufactura y la } \\
\text { distribución }\end{array}$} & $\begin{array}{l}\text { Inconsistencia del rendimiento } \\
\text { operativo }\end{array}$ \\
\hline & & Ratios inestables de producción \\
\hline & & $\begin{array}{l}\text { Alta variabilidad en los niveles de } \\
\text { inventario }\end{array}$ \\
\hline & & $\begin{array}{l}\text { Inconsistencia en la calidad de } \\
\text { los productos }\end{array}$ \\
\hline & & Tiempos inestables de entrega \\
\hline & $\begin{array}{l}\text { Relacionado con la gestión } \\
\text { de marketing }\end{array}$ & $\begin{array}{l}\text { Inestabilidad en las políticas, } \\
\text { lineamientos y estrategias de } \\
\text { marketing }\end{array}$ \\
\hline & $\begin{array}{l}\text { Relacionado con la } \\
\text { estimación }\end{array}$ & $\begin{array}{l}\text { Modelos de pronósticos } \\
\text { inadecuados }\end{array}$ \\
\hline $\begin{array}{l}\text { Origen en el } \\
\text { contexto }\end{array}$ & $\begin{array}{l}\text { Foco en las restricciones } \\
\text { de distribución }\end{array}$ & $\begin{array}{l}\text { Asignación de rutas } \\
\text { Ventanas de tiempo de entrega y } \\
\text { distribución } \\
\text { Normativas municipales } \\
\text { cambiantes }\end{array}$ \\
\hline $\begin{array}{l}\text { Origen en los } \\
\text { clientes }\end{array}$ & Foco en los clientes & Cambios atribuidos al cliente \\
\hline
\end{tabular}

Elaboración propia 
Tabla 2

Cuadrantes de abastecimiento

\begin{tabular}{|c|c|c|c|}
\hline & \multicolumn{2}{|c|}{ Incertidumbre de demanda } \\
\hline & & $\begin{array}{l}\text { Productos } \\
\text { funcionales }\end{array}$ & $\begin{array}{l}\text { Productos } \\
\text { innovadores }\end{array}$ \\
\hline \multirow{2}{*}{ 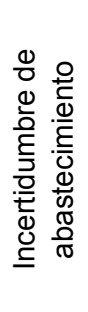 } & 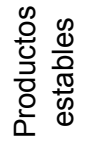 & Eficiencia & $\begin{array}{l}\text { Nivel de } \\
\text { servicio }\end{array}$ \\
\hline & 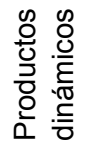 & Riesgo & Agilidad \\
\hline
\end{tabular}

Fuente: Tierney (2004), Pagano (2008)

\subsection{Gestión de demanda}

Para gestionar el riesgo se requiere tener un proceso de gestión de demanda estable y medible. La gestión de demanda es la propuesta del consumo futuro obtenido a partir de un pronóstico luego de una secuencia de consensos organizacionales, tomando en cuenta variables del mercado y restricciones de la operación comercial. Entre sus características se puede mencionar que se busca la mayor exactitud y tiene una periodicidad adecuada a cada proceso de negocio. El proceso de planificación de ventas y operaciones desarrolla el balance entre la demanda y el abastecimiento, por lo que muchos de los resultados relacionados con los riesgos de operación del negocio usualmente se identifican en dicho proceso táctico de coordinación.

Una métrica poco utilizada como herramienta táctica en la gestión de riesgo operativo de abastecimiento es la exactitud del pronóstico. Usualmente, la medición de resultados en el proceso de estimación tan importante para todo negocio tiene carácter de resultado informativo, debido a que no hay nada que hacer ante un período comercial que ya terminó. Aquí encontramos una oportunidad de utilizar este dato como un instrumento de gestión, usando la información cerrada histórica como una base de la gestión futura del abastecimiento, que en el fondo es la que influye en dicho valor. No debe sorprendernos que la exactitud de un pronóstico dependa de la naturaleza del producto (ciclo de 
vida, capilaridad en el mercado, presencia y posicionamiento frente a la competencia, etc.).

Asimismo, es posible describir su tendencia considerando la dirección del pronóstico al observar un sesgo en caso de que se mantenga de manera sistemática. Dicha tendencia puede tener un sesgo notorio en sobreestimar o subestimar, dependiendo de la estructura del sector competitivo y la madurez con la que los procesos internos del negocio se encuentren organizados.

Tabla 3

Cálculo del error del pronóstico por producto

\begin{tabular}{lcccc}
\hline & Prod 1 & Prod 2 & Prod 3 & Prod 4 \\
\hline Pronóstico & 132 & 1 & 90 & 48 \\
Real & 74 & 90 & 1 & 32 \\
Error & 58 & 89 & 89 & 16 \\
Porcentaje de error & $78 \%$ & $99 \%$ & $8900 \%$ & $50 \%$ \\
Exactitud & $22 \%$ & $1 \%$ & $0 \%$ & $50 \%$ \\
\hline
\end{tabular}

Elaboración propia

Para realizar el cálculo de una métrica de exactitud a través de un grupo de productos se puede utilizar el promedio absoluto del error porcentual (MAPE).

Secuencia:

- Sumar los valores absolutos de los errores.

- Dividir el valor total entre la cantidad total de unidades reales.

Tabla 4

Cálculo de la exactitud agregada al pronóstico

\begin{tabular}{lccccc}
\hline & Prod 1 & Prod 2 & Prod 3 & Prod 4 & Total \\
\hline Pronóstico & 23 & 48 & 5 & 40 & 116 \\
Real & 20 & 87 & 3 & 120 & 230 \\
Error & 3 & 39 & 2 & 80 & 124 \\
Porcentaje & $15 \%$ & $45 \%$ & $67 \%$ & $67 \%$ & $54 \%$ \\
de error & $85 \%$ & $55 \%$ & $33 \%$ & $33 \%$ & $46 \%$ \\
Exactitud & $85 \%$
\end{tabular}

Elaboración propia 
El MAPE es la métrica más utilizada en diversas industrias, debido a su facilidad de interpretación administrativa y de procesamiento computacional (Wagner, 2010).

El MAPE ponderado se relaciona directamente con el stock de seguridad y es bastante útil, porque de esta manera la métrica tiene relación con la gestión del abastecimiento.

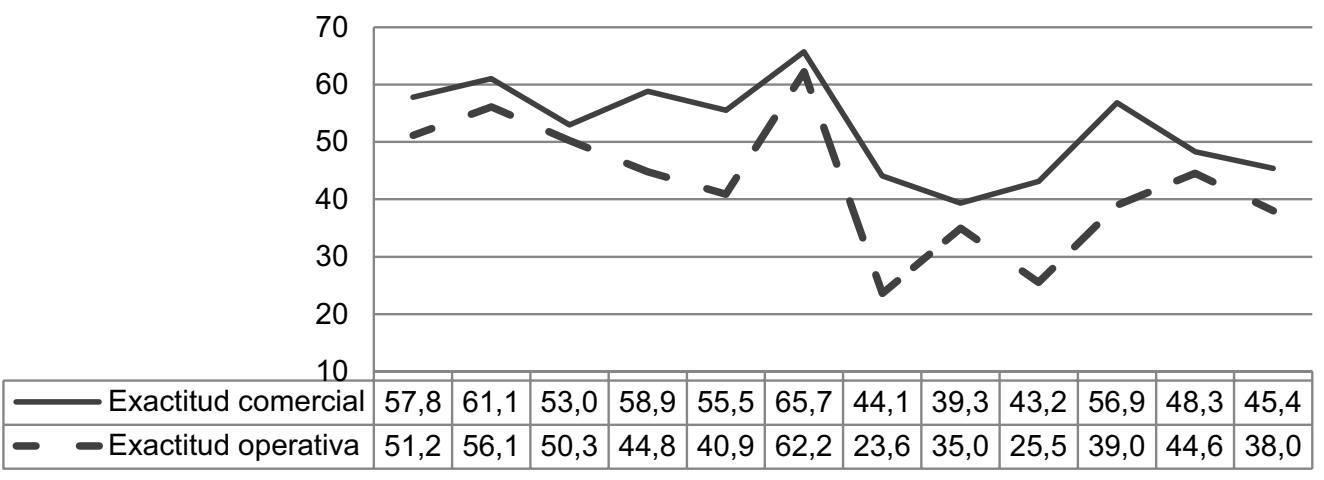

Figura 3. Seguimiento operativo de la estimación Elaboración propia

En el caso de los estimados sesgados, estos muestran la tendencia sistemática a seguir una dirección. La mayoría de los sesgos pueden ocurrir por:

- la persona que estima

- el proceso de estimación

Estos sesgos pueden ocurrir por alguna de las siguientes causas:

- Incremento del estimado para alcanzar un objetivo comercial.

- Ajuste del valor para reducir la exposición del estimado al riesgo de tener venta perdida debido a la falta de producto.

- Adición de efectos que atenúen la incertidumbre (stock de protección).

- Ciclo de vida del producto, en clara tendencia de crecimiento.

Este tipo de sesgo puede generar directamente un innecesario incremento del inventario y un mayor riesgo de productos obsoletos, si fuera el caso. 


\subsection{Tácticas para gestionar el riesgo en categorías dinámicas}

En el presente artículo sugerimos un marco de análisis que puede ser aplicado a cualquier entorno retail que tenga como característica principal gestionar productos con ciclos de vida cortos y largos tiempos de abastecimiento, lo que hace difícil predecir el volumen de demanda, generando riesgo de venta perdida o exceso de inventario. El objetivo es reducir el riesgo operativo en la cadena de abastecimiento al asignar adecuadamente políticas de inventario y modelar la demanda. Se ha encontrado que la mitigación del riesgo en el entorno retail se puede realizar utilizando diversas tácticas. Se describirá dos de ellas. La primera: controlar el riesgo por agregación, y la segunda: atenuarlo con panel de expertos ante indicios de la demanda.

\subsubsection{Control del riesgo por agregación}

En el caso del mercado retail, la agregación de los diversos skus que pertenecen a una categoría es una información útil para su gestión. Como mucho de lo que ocurre en el abastecimiento es consecuencia de negociación con proveedores para separar capacidad de producción y mantener mínimos de tamaño de lote de producción, es muy importante agregar componentes de materiales comunes a diversos productos, porque partimos del principio de que todo pronóstico agregado es más estable que un pronóstico en detalle. Esta agregación de productos también puede hacerse en función de la unidad de tiempo en que se comercializa. La disminución del riesgo de falta de componentes es una táctica para controlar el riesgo del proceso de estimación.

En todo proceso de estimación existe un grado de incertidumbre debido a las variables que se utilizan para obtener el valor esperado. Estas variables pueden ser internas o externas a la empresa. Las empresas que manufacturan y tienen como propósito el desarrollo de los proveedores buscan que el reabastecimiento ocurra en un lapso acorde con el proceso comercial. Por ese motivo, en el sector retail la necesidad de desarrollar mecanismos de integración con proveedores es una manera de compartir el riesgo de la incertidumbre de la demanda cuando se establecen contratos de separación de capacidad de manufactura.

\subsubsection{Panel de expertos ante indicios de la demanda}

En las empresas del rubro retail de productos relacionados con la moda, es muy importante ajustar la proyección de la demanda con los indi- 
cios iniciales de los consumos. Como sugieren Aksoy, Ozturk y Sucky (2012), Chase (2013), Pepe y Pepe (2012) y Fildes y Kingsman (2011), la respuesta inicial del mercado puede representar del 80 al $90 \%$ de la demanda total del período en análisis. Y en los casos de productos donde no exista una demanda histórica la estimación de los parámetros de la demanda resulta un verdadero reto para los analistas de demanda. Para esto es necesario modelar la función de demanda del producto. En productos nuevos, que no tienen parámetros históricos, se requiere trabajar con productos similares, que ayuden a tener una proyección inicial ajustada a las primeras señales de la demanda, y apoyarse en la experiencia de los gestores de demanda. Para este fin partimos del supuesto de que la diferencia entre el pronóstico y las ventas reales de productos similares es una variable aleatoria, que seguirá los mismos patrones de consumo del producto en el futuro a estimar, y evaluar la eficiencia de esos parámetros con nuevos productos proyectados para hacer el ajuste a la función de densidad. Estos parámetros también se pueden obtener de un panel de expertos, quienes dan un valor cuantitativo previamente al lanzamiento de la campaña. Los estimados del grupo experto sirven de base para obtener la media y la desviación estándar teórica inicial.

Se considera que la desviación estándar incluye un factor que busca aproximar el comportamiento histórico con el período de análisis más cercano. Es factible tener en cuenta que este factor debe variar por tipo de producto y modelo de negocio. Esto nos permite inferir que con resultados del panel más estables el valor tiende a ser más pequeño.

De manera intuitiva, se puede comprender la lógica que está en este enfoque. Usualmente, la incertidumbre en relación al valor de un estimado aparece por la variación del proceso de estimación y la variación en los parámetros de la función utilizada. Es posible pensar en tener "n" esferas de dos colores dentro de un recipiente donde se desconoce la cantidad exacta de cada color. La inquietud por saber cuántas esferas de un color específico hay en el recipiente se genera en la incertidumbre propia de un proceso con variable aleatoria discreta del tipo Bernoulli, y por no saber cuántas esferas de cada color hay en el recipiente. Si tuviéramos una opinión considerada experta, que establezca una probabilidad a priori de la cantidad de esferas de cierto color, en la medida en que esta "opinión experta" esté lejos de la realidad, la varianza de la distribución de la cantidad de esferas de cierto color también se incrementa: por lo que debe existir una correlación entre la varianza 
de la variable aleatoria número de esferas de un cierto color en un punto y la proporción de la variación en las opiniones consideradas expertas. De manera similar, en un producto considerado muy difícil de predecir para obtener la demanda, la mayor incertidumbre la genera la dispersión de la opinión individual del panel. En las empresas que desarrollan un proceso estable de estimación dicho panel puede ser consecuencia de reuniones de consenso entre diversos expertos ante un escenario comercial.

La interpretación de las opiniones de un panel de expertos en relación a un estimado futuro puede describir la probabilidad de obtener valores cercanos a una variable aleatoria. Es posible que dicha aleatoriedad varíe, por más que las condiciones del escenario sean similares (ej.: misma promoción, época del año, etc.). Dichas diferencias generan una variación que es un valor referente, ya sea porque es de un producto similar o por experiencia del negocio.

Cada opinión del experto es un resultado posible de la distribución binomial o Bernoulli. En algunas situaciones podría resultar un valor muy cercano al que ocurrirá en la realidad y en otras un valor muy alejado. La probabilidad de éxito en cada opinión es un valor constante "p". La función de probabilidad binomial está dada por la fórmula siguiente:

$$
\begin{aligned}
& f(x)=\left(\begin{array}{l}
n \\
x
\end{array}\right) p^{x}(1-p)^{n-x} \quad\left(\begin{array}{l}
n \\
x
\end{array}\right)=\frac{n !}{x !(n-x) !} \\
& x=\{0,1,2, \ldots, n\},
\end{aligned}
$$

La estimación de $\stackrel{\varpi}{i}_{i}$ puede ser entendida como estimar el valor absoluto del error del pronóstico, asumiendo que todo proceso de estimación está muy ligado a la distribución de Poisson, porque la asignación de oferta y estrategias comerciales son discretas y cambiantes en el tiempo. Para obtener la función de la distribución requerimos de una media de ocurrencia de casos de éxito y la cantidad de éxitos esperados. Se obtiene permitiendo que el tamaño de la muestra (n) se haga muy grande (tienda al infinito) y la probabilidad de éxito o de falla (p) se convierta en muy pequeña (tienda a cero) en tanto que el valor esperado (np) se mantiene constante. Por lo tanto, cuando n es grande en relación con $\mathrm{p}$, se puede utilizar la función de Poisson como una aproximación a la distribución binomial. 
Cuando tenemos una historia de errores en la medición del contexto comercial podemos considerar que esos mínimos y máximos del error de la estimación son el valor en escala de la "calidad de la estimación"; estos valores son una base de información muy útil para predecir anticipadamente el nivel de variación máximo permitido, según la historia del producto para alcanzar un nivel de exactitud esperado.

Si consideramos una variable aleatoria denominada "N", que es la diferencia histórica de las unidades entre el valor estimado previamente y el valor real obtenido, podemos considerar que dicha variación sigue una distribución Poisson, por lo que la media será el valor del producto entre el valor denominado "p" y esa variación máxima. Adicionalmente, consideramos el valor "q" como la diferencia máxima en unidades entre los paneles (llámense consensos o diferencias numéricas entre valores obtenidos cuantitativamente y los obtenidos cualitativamente). Se puede, entonces, construir una función de distribución, que nos presente la probabilidad de aceptar o rechazar un valor de proyección basado en la historia; además de ayudar a identificar el nivel de exactitud mínimo que se debería alcanzar para estar en rango factible de lograr dicha variación. La lógica se muestra en las figuras 4, 5 y 6 .

Valores de los parámetros para N: 6000 unidades, p: 0.4; y diferencia entre consenso experto de 2300 unidades.

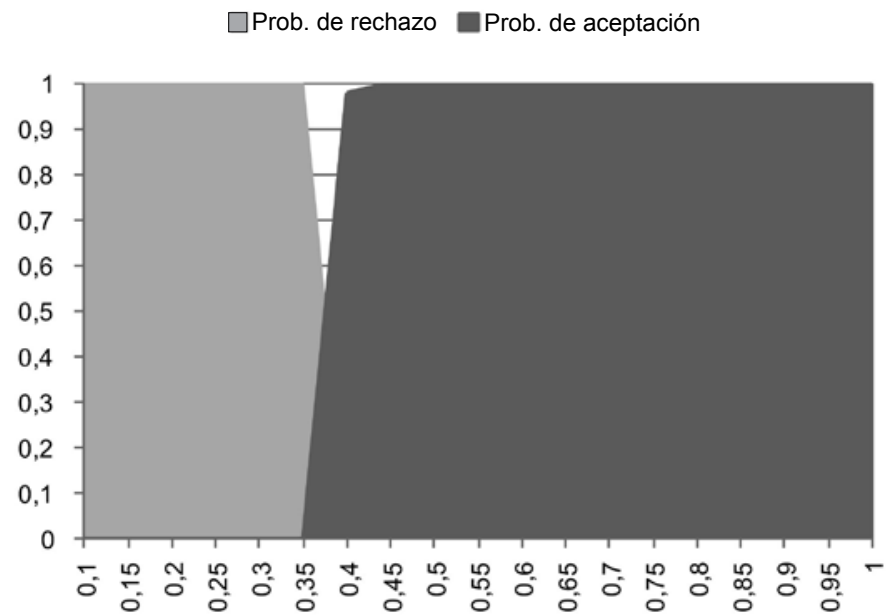

Figura 4. Resultado de decisión sobre el estimado: Delta 2300 Elaboración propia 
Valores de los parámetros para N: 6000 unidades, p: 0.4; y diferencia entre consenso experto de 1000 unidades:

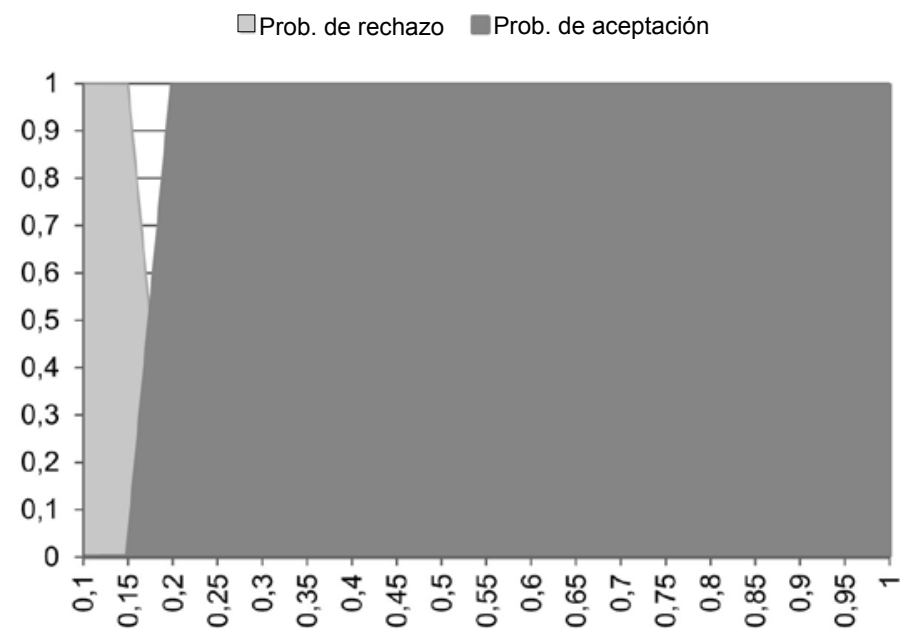

Figura 5. Resultado decisión sobre el estimado: Delta 1000 Elaboración propia

Valores de los parámetros para N: 6000 unidades, p: 0.4; y diferencia entre consenso experto de 4500 unidades:

- Prob. de rechazo $\square$ Prob. de aceptación

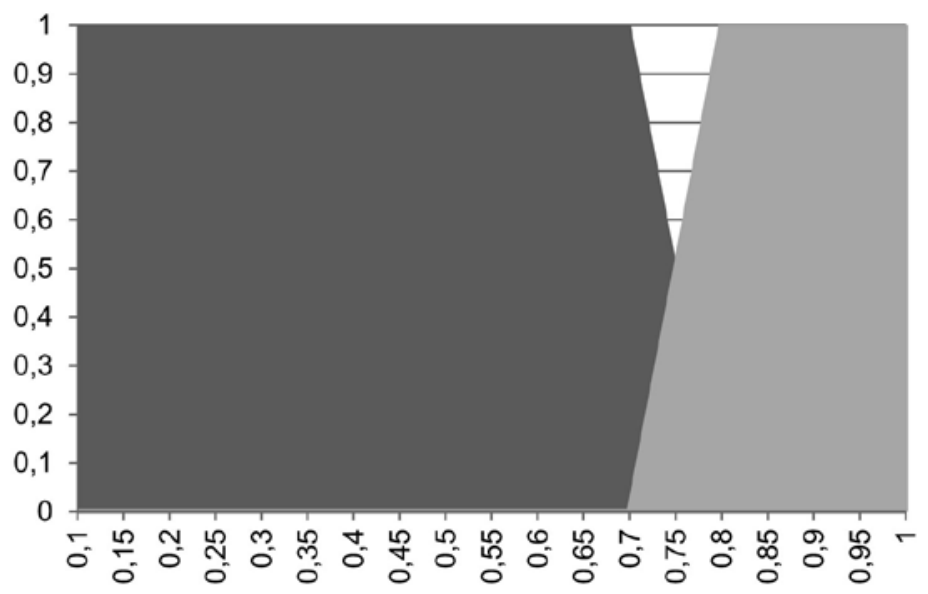

Figura 6. Resultado de decisión sobre el estimado: Delta 4500 Elaboración propia 
Como en cada negocio intervienen variables distintas, según sea el tipo de retail (no perecible, perecible, con tiendas o sin tiendas) y también enfoques de abastecimiento, según el ciclo de vida del producto, hay escenarios en donde diversos productos caen según la exactitud del estimado que tiene en diversas tácticas para mitigar el riesgo en el abastecimiento (Newbery, 2012). Considerando estos escenarios de riesgo, el seguimiento al consenso de la estimación puede ocurrir de varias maneras:
A: Prevenir y controlarlo
B: Retener el riesgo
C: Protección ante volatilidad
D: Aseguramiento de la demanda
E: Diversificación

A. En todo proceso de abastecimiento existen excepciones por diversas causas operativas. Es clave tener mapeado el riesgo de dichos efectos y desarrollar planes de contingencia para atenuar su impacto (véase la tabla 1).

B. Está relacionado con la aceptación de un riesgo, soportando las pérdidas a las que diera lugar su materialización. Incluye la aceptación de aquellos riesgos que no hayan sido identificados. En el caso de productos con promesa de entrega se puede considerar a aquellos de total satisfacción o de reembolso del monto de compra. Se utiliza mucho en la industria retail.

C. En esta estrategia se implementan mecanismos en su mayoría del tipo legal y operativo (separación de espacios y capacidad restringida), para evitar el impacto de una situación considerada de riesgo potencial. Esta estrategia se utiliza en el ámbito del retail al implementar inventarios de protección.

D. Dicha estrategia consiste en implementar un sistema de pedido que responda a un sistema de producción del tipo pull. Es menos probable que ocurran ventas perdidas en este escenario.

E. La situación de tener más de un proveedor o alternativas de reemplazo para atenuar el efecto negativo se puede representar en el ámbito comercial como toda estrategia de canjes ante falta de producto. Esto también se puede lograr desarrollando proveedores con técnicas como la planificación colaborativa (Collaborative Planning Forecasting Replenisment, CPFR), en el mercado de cosméticos se tiene 
referencia del caso L'Oreal (Beaulieu, Landry y Rebolledo, 2011) para que mejoren sus ratios de entrega y otorguen flexibilidad de respuesta. Asimismo, se considera en los casos en que el inventario se distribuye a lo largo de la cadena en componentes para evitar el exceso de producto terminado, como en el retail de arreglos florales o de productos perecibles en general.

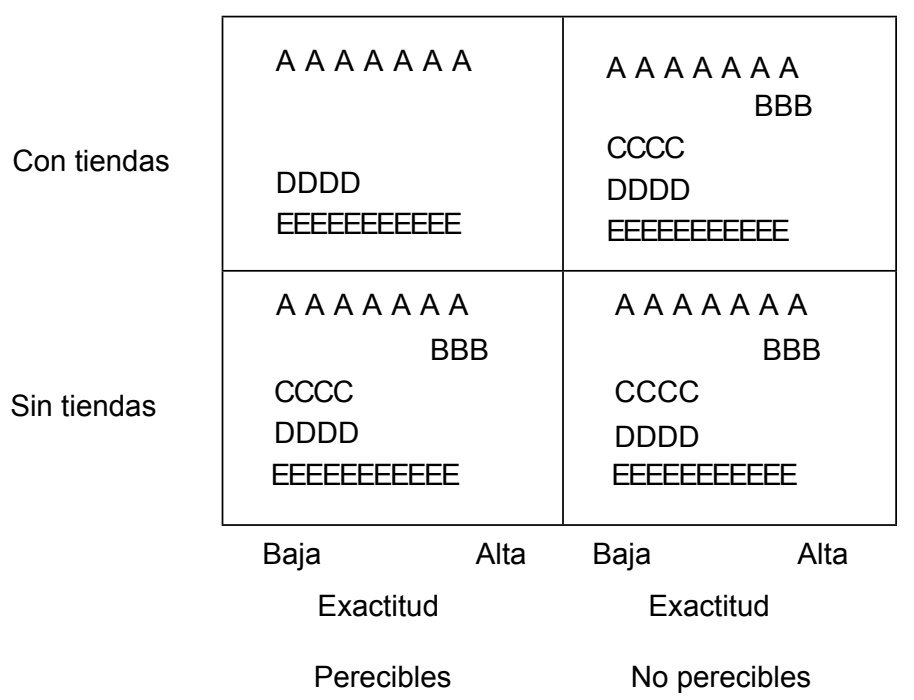

Figura 7. Matriz de interacción táctica y mitigación de riesgo Elaboración propia

La incertidumbre de estos parámetros es parte de todo proceso comercial. La aleatoriedad en la elección de un producto en particular (por ejemplo, color y talla en una prenda de vestir por parte del cliente) y en calcular la función de variación de dicha demanda por el lado comercial, ayudará a establecer límites a la variación en función del riesgo de perder venta.

Por eso, el nivel de variación puede darse por una desviación estándar predefinida o también obtenida del comportamiento promedio de la variación de los productos similares.

\section{CONCLUSIONES}

Para los promotores de proyectos retail en San Juan de Lurigancho una variable muy relevante para identificar una zona potencial de ubi- 
cación de un supermercado o un local comercial es la densidad poblacional. Actualmente existen oportunidades de desarrollo de este canal en dicho distrito, pero las dificultades para la formalización de terrenos y un perfil del consumidor que tiende más al canal tradicional impiden un crecimiento mayor.

En relación con la oferta de productos y su gestión, considerar el precio en el contexto ceteris paribus de la mezcla de variables que hacen la venta en un negocio retail es un supuesto que depende del enfoque que la empresa desea darle para competir en el mercado. Los análisis de elasticidad basados en el precio-producto utilizando información histórica pueden verse afectados por la existencia de la venta intraproductos dentro de la misma categoría, por lo que el balance del portafolio gestionando las curvas de ciclo de vida son la mejor herramienta de análisis táctico y operativo.

A diferencia de otros procesos organizacionales más estandarizados, como compras, gestión de activos, cierre contable, por citar algunos, la gestión comercial es tan particular en cada empresa por lo que no se ha identificado un software integrado que cubra las dimensiones de la gestión de una categoría, sino aplicativos a medida desarrollados muchas veces internamente para describir los procesos de decisión del negocio.

La existencia de una ley de dinero electrónico en el Perú, una de las pocas en la región, es una oportunidad para las instituciones académicas y empresariales de promover un mayor esfuerzo por innovar o desarrollar el sector comercial retail vía internet.

El riesgo de reducir el alcance de la gestión de la categoría y no integrarlo con el proceso de abastecimiento es alto. Es común que esta falta de integración genere consecuencias en el desarrollo de ambos procesos.

La visión de la categoría está orientada a atenuar o reducir la falta de inventario, gestionando el valor con el volumen disponible, mientras que la gestión de demanda se orienta a ofrecer el mayor nivel de servicio en volumen o unidades. Existen diferencias de alcance y de inicio y fin de procesos.

La estimación de productos innovadores o relacionados con la moda tienen una complejidad alta y una variabilidad en la demanda difícil de predecir, generando un reto a la cadena de abastecimiento; esto se puede atenuar aplicando técnicas de mitigación del riesgo, como las descritas en el presente artículo. Es importante que una organización reconozca 
que en ella conviven diferentes cadenas de abastecimiento orientadas a atender diversas categorías, y esto debe crear diferentes matrices de gestión de riesgo, considerando tácticas propias de cada categoría.

\section{REFERENCIAS}

Aksoy, A., Ozturk, N., y Sucky, E. (2012). A decision support system for demand forecasting in the clothing industry. International Journal of Clothing Science and Technology, 24(4), 221-236.

Beaulieu, M., Landry, S., y Rebolledo, C. (2011). L'expérience CPFR de L'Oréal Canada: la réalité au quotidien (B) (pp. 1-9). Montreal: HEC Montreal.

Chase, C. W. (2013). Using demand sensing and shaping to improve demand forecasting. The Journal of Business Forecasting, 32(4), 24-28, 31.

Crespo, P. (2015). El crecimiento retail en Casa \& Ideas. G de Gestión, 51, 27.

Díaz, M. A. (2000). Gestión por categorías y trade marketing. Madrid: Prentice Hall.

Fildes, R., y Kingsman, B. (2011). Incorporating demand uncertainty and forecast error in supply chain planning models. The Journal of the Operational Research Society, 62(3), 483-500.

Fisher, M., Kumar, R., y Raman, A. (2001). Optimizing inventory replenishment of retail fashion products. Manufacturing \& Service Operations Management, 3(3), 230-241.

García, V. E. (2011). Una aproximación al retail moderno. Lima: Universidad del Pacífico.

Gaur, V., Kesavan, S., Raman, A., y Fisher, M. L. (2007). Estimating demand uncertainty using judgmental forecasts. Manufacturing \& Service Operations Management, 9(4), 480-491.

Ipsos Apoyo Opinión y Mercado (2013). Informe gerencial de marketing: perfil del ama de casa. Lima.

Jeyaraj, A., y Sabherwal, R. (2014). The bass model of diffusion: recommendations for use in information systems research and practice. JITTA: Journal of Information Technology Theory and Application, 15(1), 5-30. 
Kurtulus, M., y Toktay, B. (2012). The impact of limited retail shelf space on category management. SSRN Working Paper Series.

Labajo, G. V. (2007). Trade marketing: la gestión eficiente de las relaciones entre fabricante y distribuidor. Madrid: Pirámide.

Newbery, M. (2012). Risk analysis of the apparel supply chain in 20122012 edition: risks in the supply chain (pp. 23-28). Bromsgrove, United Kingdom.

Ntzoufras, I. (2011). Bayesian modeling using WinBUGS (Vol. 698). New York: John Wiley \& Sons.

Pagano, A. (2008). Building supply chain excellence in emerging economies. En Hau L. Lee, Chung-Yee Lee (Eds.). New York: Springer.

Pepe, M. R., y Pepe, M. S. (2012). Using point of sale (POS) data to deliver customer value in the supermarket industry through category management practices. Journal of Marketing Development and Competitiveness, 6(1), 69-73.

Samardzich, C. (2014). Lima de aniversario: los problemas urbanísticos de la capital. Semana Económica. Recuperado de http:// semanaeconomica.com/article/servicios/130821-lima-deaniversario-los-problemas-urbanisticos-de-la-capital/

Singh, J., y Raghuvanshi, R. (2014). Role of supply chain management in retail sector. International Journal of Management Research and Reviews, 4(11), 1091-1103.

Tierney, S. (2004). The causes of complexity. Supply Chain Europe, 13(3), 20-21.

Valdiviezo, C. (2015). Comercio electrónico en el Perú: ¿será éste el año del despegue definitivo? Semana Económica. Recuperado de http://semanaeconomica.com/article/servicios/154181-comercioelectronico-en-el-peru-sera-este-el-ano-del-despegue-definitivo/

Wagner, M. (2010). Forecasting daily demand in cash supply chains. American Journal of Economics and Business Administration, 2(4), 377-383.

Wieland, A. (2013). Selecting the right supply chain based on risks. Journal of Manufacturing Technology Management, 24(5), 652-668. 
